\title{
Temperature Monitoring during Focused Ultrasound Treatment by Means of the Homodyned $\boldsymbol{K}$ Distribution
}

\author{
M. Byra*, E. Kruglenko, B. Gambin and A. Nowicki \\ Department of Ultrasound, Institute of Fundamental Technological Research, Polish Academy of Sciences, \\ A. Pawińskiego 5B, 02-106, Warsaw, Poland \\ (Received October 3, 2016; in final form May 19, 2017)

\begin{abstract}
Temperature monitoring is essential for various medical treatments. In this work, we investigate the impact A tissue mimicking phantom was heated with a spherical ultrasonic transducer up to $56^{\circ} \mathrm{C}$ in order to imitate tissue necrosis. During the heating, an imaging scanner was used to acquire backscattered echoes from the heated region. These data was then modeled with the homodyned $K$ distribution. We found that the best temperature indicator can be obtained by combining two parameters of the model, namely the backscattered echo mean intensity and the effective number of scatterers per resolution cell. Next, ultrasonic thermometer was designed and used to create a map of the temperature induced within the tissue phantom during the treatment.
\end{abstract} \\ of temperature on backscattered ultrasound echo statistics during a high intensity focused ultrasound treatment.
}

DOI: 10.12693/APhysPolA.131.1525

PACS/topics: temperature monitoring, homodyned $K$ distribution, focused ultrasound

\section{Introduction}

There are several medical treatments for which temperature monitoring is necessary. First, in the case of hyperthermia temperature is usually maintained between $39^{\circ} \mathrm{C}$ and $42^{\circ} \mathrm{C}$ to induce physiological changes beneficial to patient's health [1]. This temperature range guarantees that all living functions of cells will be preserved. Second, high intensity focused ultrasound (HIFU) is a tool which can be used to heat tissues $\left(>56^{\circ} \mathrm{C}\right)$ and induce necrosis in the target volume. HIFU has been applied for treating cancer [2,3] and uterine fibroids [4].

Various methods have been proposed to monitor temperature during tissue heating [5]. Up to date, magnetic resonance imaging (MRI) is considered the best for temperature monitoring. However, this technique of imaging is complex and expensive which limit the range of applications. On the other hand, ultrasound (US) systems were proposed both for imaging and therapy. These systems are more robust and far less expensive than MRI. To monitor temperature variations, several US-based methods were proposed. First of all, the change in signal's backscattered energy (CBE) was reported as a good temperate indicator in several studies [6-8]. CBE is based on the fact that heating modifies acoustic impedance of the tissue and subsequently the reflectivity [9]. This phenomenon can be clearly observed on the B-mode image where the heated area is usually brighter. Tsui et al. $[10,11]$ proposed to model the statistics of backscattered echo amplitudes with the Nakagami distribution [12] and to use the Nakagami parameter for temperature monitoring. Gambin and Kruglenko investigated the usefulness of other distributions such as the $K$ and the Rayleigh distribution [13]. Additionally, Byra and Gambin

*corresponding author; e-mail: mbyra@ippt.pan.pl proposed to assess temperature dependent variations in backscattered echo statistics by means of nonparametric methods [14].

In this work we use the homodyned $K$ distribution for temperature monitoring. This model describes the statistics of backscattered echo with three parameters and is considered to be highly effective in scattering properties characterization [15]. The homodyned $K$ distribution was reported to be useful for tissue characterization [16], especially in the case of breast lesion classification $[17,18]$. Parameters of the homodyned $K$ distribution describe different aspects of the acoustic scattering, namely the mean intensity of backscattered echo, number of scatterers per resolution cell and their spatial organization. In this work we decided to use all distribution parameters. This is in contrast to studies which utilizes the mean intensity or scale-free shape parameters (e.g. Nakagami parameter) only. We expect that by means of parameter combination a better temperature estimation may be obtained. The homodyned $K$ distribution gives more detailed scattering description than the Nakagami distribution. Therefore, by means of the homodyned $K$ distribution it is possible to better understand the physical foundations of temperature dependent variations in backscattered echo statistics. We conducted an experiment consisting a tissue phantom which was heated up to $56^{\circ} \mathrm{C}$ in order to imitate necrosis induction during HIFU treatment.

This paper is organized as follows, first we describe the experimental setup and methods of temperature assessment by means of homodyned $K$ distribution. Next, results are presented and discussed.

\section{Materials and methods 2.1. Experimental setup}

Tissue phantom was made out of agar powder dissolved in water. The detailed recipe can be found in [19]. Glass microparticles (model 59200-U, Supelco, Bellefonte, PA, 
USA, diameter $75 \pm 5 \mu \mathrm{m}$ ) were added to the agarwater solution in order to imitate tissue microstructure. Phantom had cylindrical shape of diameter and height of $5 \mathrm{~cm}$ and $9 \mathrm{~cm}$, respectively.

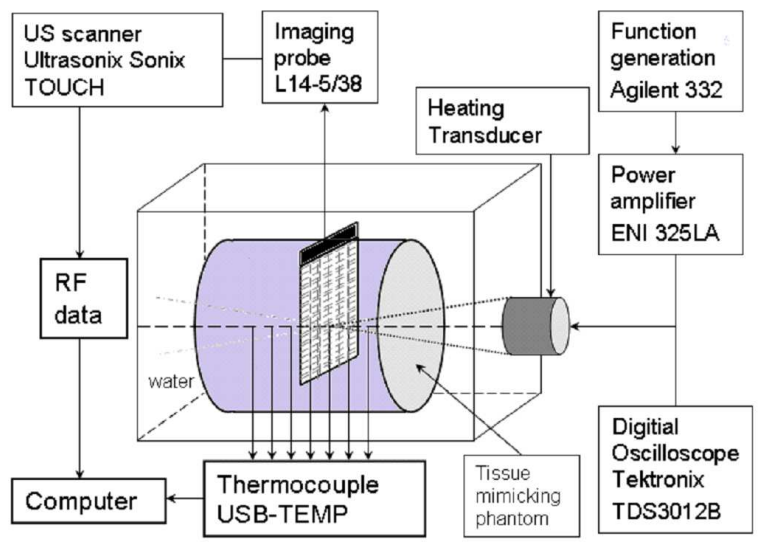

Fig. 1. Experimental setup used for tissue phantom heating.

The sample was positioned in a tank filled with temperature-controlled degassed distilled water [20, 21]. Figure 1 shows the experimental setup which includes a generator (Agilent 332, Aprings Colorado, USA), an amplifier (ENI 1325LA, Rochester NY, USA), and an oscilloscope (Tektronix TDS3012B). Spherical ultrasonic transducer (central frequency $2.2 \mathrm{MHz}$, diameter $44 \mathrm{~mm}$ and $44.5 \mathrm{~mm}$ focal length) was used to heat the sample. The average power of focused ultrasound beam was $6 \mathrm{~W}$. The focal zone was set at a depth of $30 \mathrm{~mm}$ within the phantom. A more comprehensive description of the experimental setup can be found in [20,21].

The experiment was performed in two steps. First, the sample was heated and the spatio-temporal temperature variations were registered. In the second step, the same heating process was repeated and simultaneously RF signals were collected. During $10 \mathrm{~min}$ of heating temperature was recorded with thermocouples and registered by the module USB-TEMP (Measurement Computing, Norton, USA). The temperature within the phantom was increased up to $56^{\circ} \mathrm{C}$. Ultrasound scanner (Sonix TOUCH, Ultrasonix, British Columbia, Canada) was used to store raw RF images (pre-beamformed) every $10 \mathrm{~s}$ during the heating. Linear imaging probe (L14-5/38) was located above the focal zone which was determined as the place where the highest temperature increase was recorded with the thermocouples. Additionally, the imaging plane was transverse to the heating beam axis as it is depicted in Fig. 1. The sampling rate and the imaging frequency were $40 \mathrm{MHz}$ and $10 \mathrm{MHz}$, respectively.

\subsection{Backscattered echo assessment}

The homodyned $K$ distribution of the backscattered echo envelope can be expressed in terms of an improper integral as follows [22]:

$$
p(A)=A \int_{0}^{\infty} x J_{0}(s x) J_{0}(A x)\left(1+\frac{x^{2} \sigma^{2}}{2 u}\right)^{-u} \mathrm{~d} x,
$$

where $A$ stands for the amplitude, $s^{2}$ and $\sigma^{2}$ are the coherent and diffuse backscattered energy, respectively. $J_{0}$ is the zero-order Bessel function of the first kind. The homodyned $K$ distribution is characterized with three parameters. The scattering clustering parameter $u$ refers to the number of scatterers per resolution cell. The derived parameter $k=\frac{s}{\sigma}$ describes the level of periodicity in scatterer distribution. Additionally, $I=E\left[A^{2}\right]$ is the mean intensity of backscattered echo. We used the level set algorithm to estimate $u$ and $k$ parameters from envelope samples [23].

Statistical parameters for spatial regions can be calculated in various ways [24-26]. Maps of the homodyned $K$ distribution parameters were calculated for each RF data image with the sliding-window technique [25]. Square window of size $2 \mathrm{~mm}$ was moved in steps of one pixel. In this case, size of $2 \mathrm{~mm}$ corresponded to approximately 3 pulse lengths. This choice was reported as optimal in studies on Nakagami imaging [25, 27].

\section{Results}

Figure 2 compares two B-mode images reconstructed from RF data acquired before heating and in the moment of maximum heat. As it can be observed, due to heating the second B-mode image is slightly brighter and the speckle pattern had changed. Here, focal zone was indicated with a black circle. Temperature variations in the focal zone measured with the thermocouple are depicted in Fig. 3.
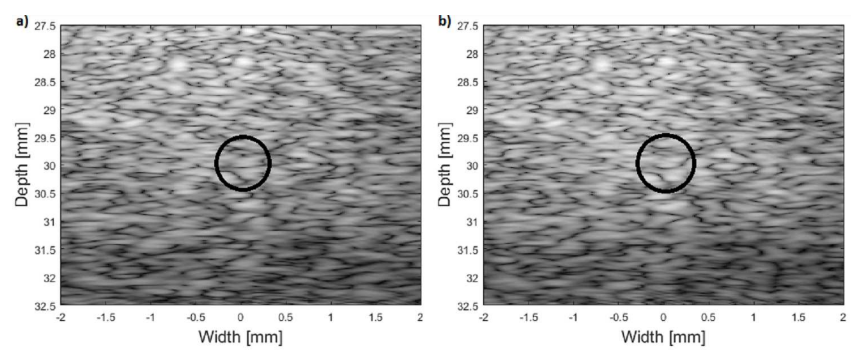

Fig. 2. Phantom B-mode images: (a) before heating, (b) moment of maximum heat. Focal zone indicated with a circle.

For each RF data matrix envelopes were calculated with the Hilbert transform and parametric maps were created with the sliding window technique. All calculations were carried out in Matlab (Mathwork Inc., Natick, MA). Maps values within the radial ROI (decimeter of $1 \mathrm{~mm}$, see Fig. 2) were averaged. These averages were considered to be representative for the focal zone. Next, obtained values of parameters were used to perform regression which is shown in Fig. 4. Parameter values in moment zero were subtracted. Additionally, intensity 


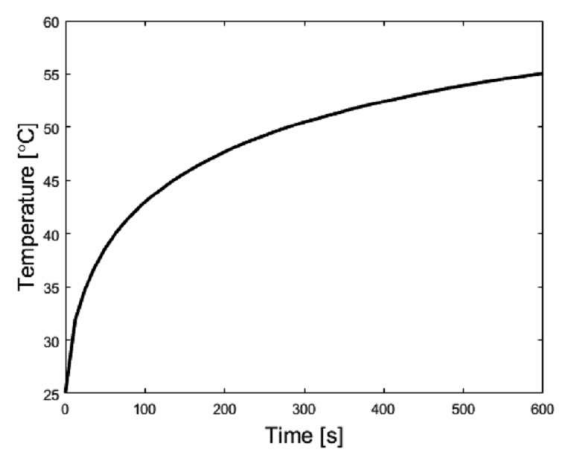

Fig. 3. Temperature measured in the focal zone with the thermocouple.

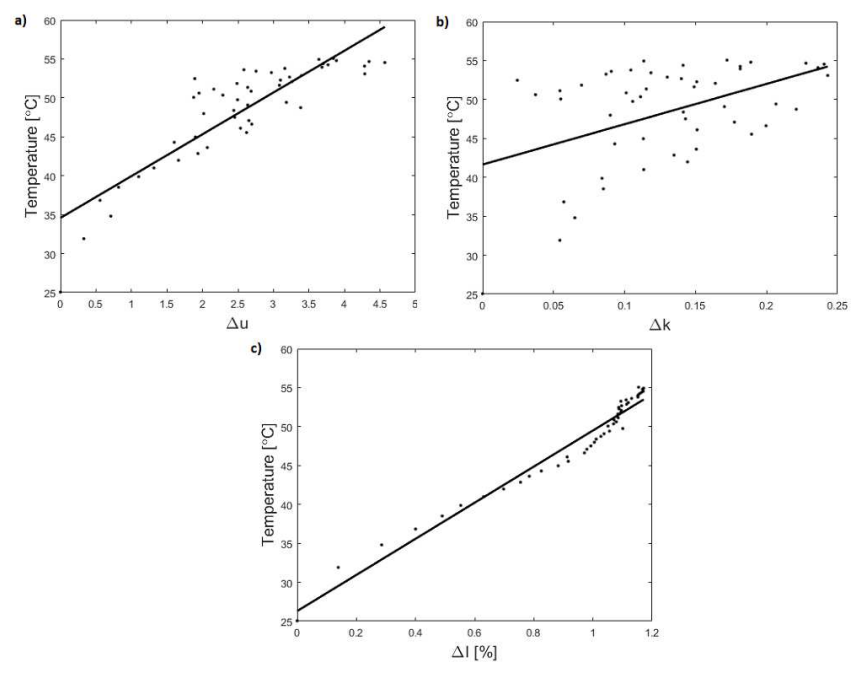

Fig. 4. Temperature as function of the homodyned $K$ distribution parameters.

was normalized to omit its high values. Parameters of the best linear models are presented in Table I.

TABLE I

Linear regression results for graphs in Fig. 4.

\begin{tabular}{c|c|c|c}
\hline \hline Parameter & Slope $( \pm$ std $)$ & Intercept $( \pm$ std $)$ & $R^{2}$ \\
\hline$\Delta u$ & $5.3724 \pm 0.4303$ & $34.576 \pm 1.197$ & 0.785 \\
$\Delta k$ & $51.845 \pm 14.397$ & $41.638 \pm 2.0533$ & 0.213 \\
$\Delta I$ & $23.209 \pm 0.65989$ & $26.297 \pm 0.65401$ & 0.913 \\
mc4c & & &
\end{tabular}

TABLE II

The best linear model for temperature.

\begin{tabular}{c|c}
\hline \hline Parameter & Value $( \pm$ std $)$ \\
\hline Intercept & $26.764 \pm 0.63767$ \\
$\Delta u$ slope & $0.84105 \pm 0.30866$ \\
$\Delta I$ slope & $20.446 \pm 1.1884$ \\
$R^{2}$ & 0.978
\end{tabular}

Next, we performed a search for a linear model of parameters which have the highest value of $R^{2}$. The best fit was obtained with two parameters, namely $u$ and $I$. Results are depicted in Table II.

The best linear model was used as a temperature indicator. Figure 5 shows how temperature would be estimated in the focal zone with the proposed model during heating. Additionally, our model was used to create a map of temperature (Fig. 6) for the B-mode image from Fig. 2b.

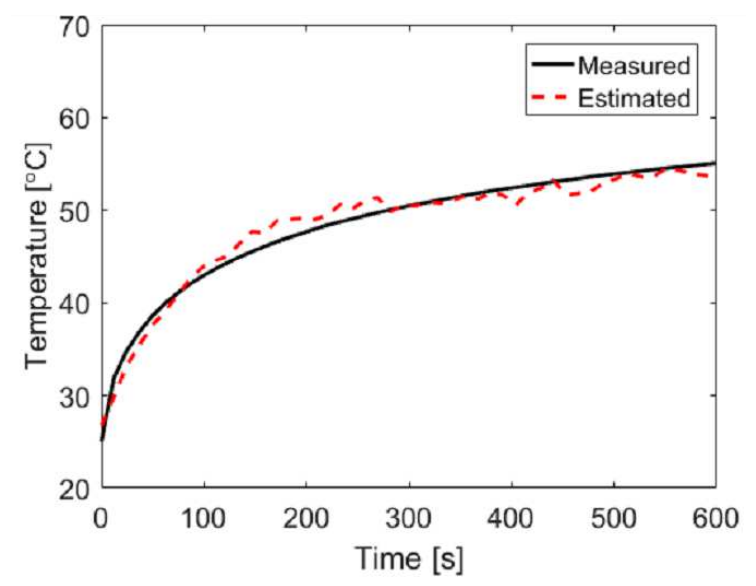

Fig. 5. Comparison of thermometer and temperature measured by the thermocouple.

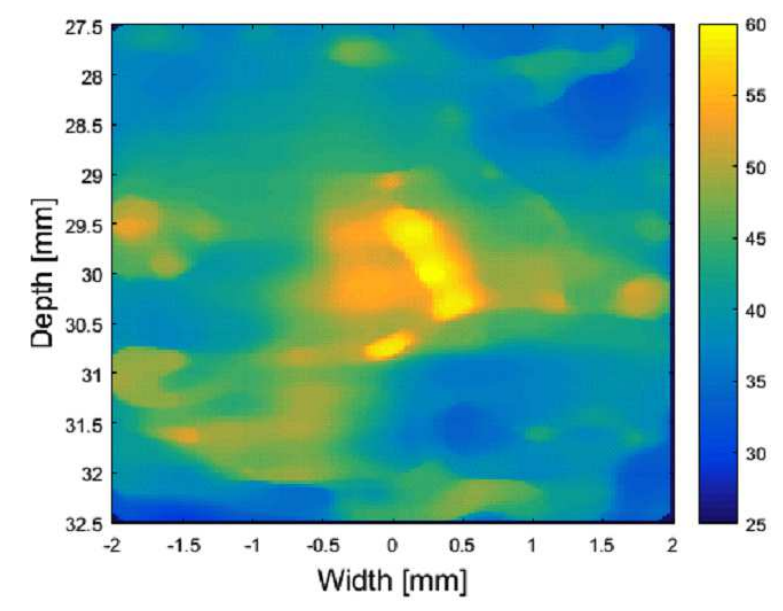

Fig. 6. Temperature map for B-mode image from Fig. $2 b$.

\section{Discussion and conclusions}

According to Table II, best temperature indicator was obtained using two parameters, namely the intensity $I$ and the effective number of scatterers per resolution cell $u$. Clustering parameter $k$ showed to be not useful. This parameter had low value of $R^{2}$ and could not improve the linear model consisting the remaining two parameters.

Temperature monitoring by means of intensity was reported to be effective in several works [6-8]. In our case, intensity was the best individual parameter. In comparison, the $u$ parameter was slightly worse. The change of 
intensity and $u$ due to heating has different physical explanation. In the case of intensity heating modifies local tissue reflectivity [9]. This phenomenon directly leads to a change in intensity of backscattered echo. The impact of heating on the remaining homodyned $K$ distribution parameters is, however, more complex. With the temperature increase size of resolution cell is modified. This change is due to tissue thermal expansion and temperature dependent speed of sound [28]. According to Fig. 4a in the case of our tissue phantom, the size of the resolution cell increased with temperature. The derived parameter $k$ is the ratio of coherent to diffuse backscattered energy. This parameter was less affected by the temperature in our case. Supposedly heating had similar impact on both the coherent and the diffuse energy. On the other hand, since the phantom was augmented with randomly distributed microparticles, the diffuse scattering was predominating resulting in the overall irrelevance of the $k$ parameter.

Figure 3 shows the temperature field in the moment of maximum heat. The highest temperature was indicated in the focal zone, as expected. However, the effect of heating was also visible outside the focal zone. This was probably caused by a relatively long time of heating which resulted in energy dissipation.

To the best of our knowledge, the intensity and statistical parameters were not combined in literature so far. In our work, we showed that such combination leads to a better temperature monitoring. Although good results were obtained in our study in the case of a tissue phantom, it must be pointed out that a separate calibration should be necessary for another material. Our method may be especially useful in monitoring temperature induced in tissues and phantoms which were enriched with nanoparticles [29]. However, estimated parameters depend on tissue coefficients such as the thermal expansion or the acoustic impedance.

The homodyned $K$ distribution proved to be useful in our case. This model describes a wider range of scattering scenarios than the Nakagami distribution. Therefore, it can be used for a wider range of tissues, especially for the scattering scenarios which cannot be modeled by the Nakagami distribution.

\section{Acknowledgments}

This work was supported by the National Science Center Grant number UMO-2014/13/B/ST7/01271.

\section{References}

[1] J. Foiret, K.W. Ferrara, PLoS One 10, (2015).

[2] L. Poissonnier, J.Y. Chapelon, O. Rouvière, L. Curiel, R. Bouvier, X. Martin, J.M. Dubernard, A. Gelet, Eur. Urol. 51, 381 (2007).

[3] J. Kennedy, Nature Rev. Canc. 5, 321 (2005).

[4] E.A. Stewart, J. Rabinovici, C.M.C. Tempany, Y. Inbar, L. Regan, B. Gastout, G. Hesley, H.S. Kim, S. Hengst, W.M. Gedroye, Fertil. Steril. 85, 22 (2006).
[5] M.A. Lewis, R.M. Staruch, R. Chopra, Int. J. Hyperth. 31, 163 (2015).

[6] J.W. Trobaugh, R.M. Arthur, W.L. Straube, E.G. Moros, Ultrasound Med. Biol. 34, 289 (2008).

[7] J. Xia, Q. Li, H.L. Liu, W.S. Chen, P.H. Tsui, Comput. Math. Methods Med. 2013, 682827 (2013).

[8] X. Li, G. Ghoshal, R.J. Lavarello, M.L. Oelze, Med. Phys. 52901, 51 (2014).

[9] R.M. Arthur, J.W. Trobaugh, W.L. Straube, E.G. Moros, IEEE Trans. Ultrason. Ferroelectr. Freq. Control 52, 1644 (2005).

[10] Z. Zhou, S. Wu, C.Y. Wang, H.Y. Ma, C.C. Lin, P.H. Tsui, PLoS One 10, (2015).

[11] P.-H. Tsui, Y.-C. Shu, W.-S. Chen, H.-L. Liu, I.-T. Hsiao, Y.-T. Chien, Med. Phys. 39, 2369 (2012).

[12] P.M. Shankar, IEEE Trans. Ultrason. Ferroelectr. Freq. Control 47, 727 (2000).

[13] B. Gambin, E. Kruglenko, Acta Phys. Pol. A 128, A-72 (2015).

[14] M. Byra, B. Gambin, Hydroacoustics 18, 17 (2015).

[15] F. Destrempes, G. Cloutier, Ultrasound Med. Biol. 36, 1037 (2010).

[16] M.L. Oelze, J. Mamou, IEEE Trans. Ultrason. Ferroelectr. Freq. Control 63, 336 (2016).

[17] M. Byra, A. Nowicki, H. Wróblewska-Piotrzkowska, K. Dobruch-Sobczak, Med. Phys. 43, 5561 (2016).

[18] I. Trop, F. Destrempes, M. El Khoury, A. Robidoux, L. Gaboury, L. Allard, B. Chayer, G. Cloutier, Radiology 275, 666 (2015).

[19] E. Kruglenko, B. Gambin, L. Cieślik, Hydroacoustics 16, 121 (2013).

[20] B. Gambin, E. Kruglenko, T. Kujawska, M. Michajłow, Acta Phys. Pol. A 119, 950 (2011).

[21] T. Kujawska, W. Secomski, M. Byra, M. Postema, A. Nowicki, Ultrasonics 76 (2017).

[22] E. Jakeman, R.J.A. Tough, J. Opt. Soc. Am. A 4, 1764 (1987).

[23] D.P. Hruska, M.L. Oelze, IEEE Trans. Ultrason. Ferroelectr. Freq. Control 56, 2471 (2009).

[24] A. Nowicki, H. Piotrzkowska-Wroblewska, J. Litniewski, M. Byra, B. Gambin, E. Kruglenko, K. DobruchSobczak, in: 2015 IEEE Int. Ultrason. Symp. (IUS), Taipei (Taiwan), 2015, art. 417.

[25] P.-H. Tsui, C.-C. Chang, Ultrasound Med. Biol. 33 , 608 (2007).

[26] P.M. Shankar, V.A. Dumane, J.M. Reid, V. Genis, F. Forsberg, C.W. Piccoli, B.B. Goldberg, IEEE Trans. Ultrason. Ferroelectr. Freq. Control 48, 569 (2001).

[27] M. Byra, A. Nowicki, H. Piotrzkowska-Wroblewska, J. Litniewski, K. Dobruch-Sobczak, in: 2015 IEEE Int. Ultrason. Symp. (IUS), Taipei (Taiwan), 2015, art. 408.

[28] C. Simon, P. VanBaren, E.S. Ebbini, IEEE Trans. Ultrason. Ferroelectr. Freq. Control 45, 1088 (1998).

[29] A. Józefczak, K. Kaczmarek, T. Hornowski, M. Kubovcikowa, Z. Rozynek, M. Timko, A. Skumiel, Appl. Phys. Lett. 108, 263701 (2016). 